\title{
B-Physics at the LHC
}

\author{
V. V. Gligorov ${ }^{\text {a* }}$ \\ ${ }^{\mathrm{a} U n i v e r s i t y ~ o f ~ G l a s g o w, ~ G l a s g o w, ~ G 12 ~ 8 Q Q, ~ U K ~}$
}

Data which will be taken at the Large Hadron Collider (LHC) will allow a rich programme of B-physics studies to be carried out, both at LHCb, LHC's dedicated heavy flavour experiment, and at ATLAS and CMS. The aim of these studies is to over-constrain the CKM matrix in order to search for New Physics in the flavour sector. This contribution gives an overview of the B-physics programme at the LHC. The detectors are introduced in the context of the LHC's likely 2009 running conditions, and certain outstanding issues in B-physics are reviewed. Four areas of the LHC's B-physics programme are looked at in more detail: measurements of rare decay processes $B_{s}^{0} \rightarrow \mu^{+} \mu^{-}$and $B_{d}^{0} \rightarrow K^{* 0} \mu^{+} \mu^{-}$; measurements of the CKM angle $\gamma$; measurements of the $B_{s}^{0}$ mixing phase $\phi_{s}$; and measurements of radiative penguin decays $B^{0} \rightarrow s \gamma$.

\section{Introduction}

It is a historical reality that discoveries of anomalies in quantum loop processes have often preceded the discovery of new physics at the energy frontier, because quantum loop processes probe energy scales inaccessible to direct searches. As an example, the discovery of $\mathrm{CP}$ violation lead Kobayashi and Maskawa to predict the existence of the third quark generation long before it would be directly discovered. And even in the era of the Large Hadron Collider (LHC), flavour studies will remain necessary to fully understand any new physics which may be discovered. Indeed, if no new particles are discovered at the LHC, flavour physics will still be able to probe their effects at scales far out of reach of direct searches; and if new particles are seen, flavour physics provides complementary information to constrain new physics models. In this Section the reader will be briefly reminded of our current knowledge of $\mathrm{CP}$ violation, and the status of the LHC and its detectors, before the subsequent Sections outline some of the flavour physics measurements which will be carried out at the LHC.

\subsection{Current knowledge of $\mathrm{CP}$ violation}

Knowledge of the CKM matrix, which describes CP violation in the Standard Model (SM),

\footnotetext{
* Now at CERN, Switzerland. Work presented on behalf
} of the LHCb collaboration. has improved significantly in recent and all the $\mathrm{CP}$ conserving and violating measurements provide a rather consistent picture that flavour mixing can be described by the 3 by 3 unitary CKM matrix with four independent parameters. However, in order to over-constrain the parameters, several measurements still need to be improved. Chief among these are the angle $\gamma$, one of the angles of the Unitarity Triangle, and the $B_{s}^{0}$ mixing phase $\phi_{s}$. The CKMFitter [1] average of $\gamma$ from direct measurements is

$\gamma=76.8^{\circ}[+27-30]^{\circ}$,

which can be compared to the global SM constraint

$\gamma=67.6^{\circ}[+4.2-3.9]^{\circ}$.

While $\phi_{s}$ is predicted to be very small in the SM

$\phi_{s}=-0.0368 \pm 0.0018 \mathrm{rad}$

recent measurements at $\mathrm{CDF}$ and $\mathrm{D} 0$ [2] have indicated a degree of tension with this prediction, as can be seen in Figure 1, reproduced from the CKMFitter website. Making precision measurements of these parameters is a key goal of the flavour physics programme at the LHC, as any deviation from their SM values would be a clear sign of NP. 


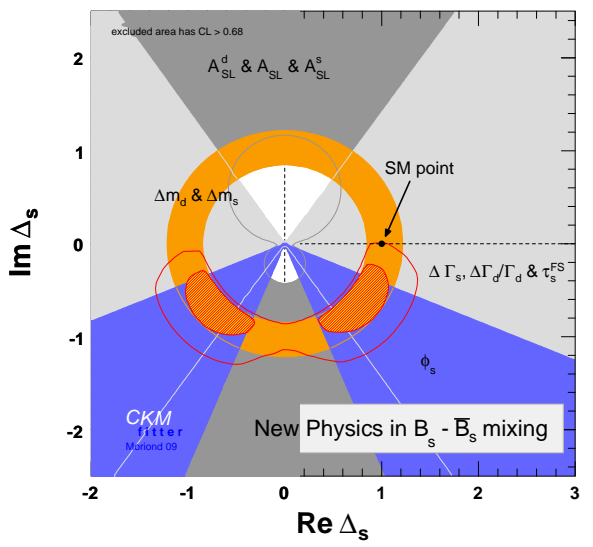

Figure 1. Current experimental constraints on $B_{s}^{0}$ mixing with the Standard Model prediction indicated [1].

\subsection{The Large Hadron Collider experi- ments: ATLAS, CMS, and LHCb}

Based at CERN, the LHC [3] is a protonproton collider which will operate at a centre-ofmass energy of $14 \mathrm{TeV}$, with a maximum luminosity of $10^{34} \mathrm{~cm}^{-2} \mathrm{~s}^{-1}$. At design parameters the LHC will collide proton bunches with a frequency of $40 \mathrm{MHz}$. There are many detectors placed along the LHC's circumference, but three are relevant for the physics topic and specifically considered in this contribution: the two general purpose detectors, ATLAS and CMS, and the LHC's dedicated heavy-flavour detector, LHCb.

ATLAS and CMS $[4,5]$ are both hermetic general purpose detectors, with a design luminosity of $10^{34} \mathrm{~cm}^{-2} \mathrm{~s}^{-1}$ and full coverage up to a pseudorapidity $(\eta)$ of 2.4. Both intend to carry out some B-physics studies while running at a luminosity of $10^{33} \mathrm{~cm}^{-2} \mathrm{~s}^{-1}$ during the beginning of LHC operation, collecting $30 \mathrm{fb}^{-1}$ of data. Both experiments are designed to collect data at a rate of $100 \mathrm{~Hz}$, which should be compared to the bunch crossing rate of $40 \mathrm{MHz}$. In order to reduce the one to the other, ATLAS and CMS will trigger on high transverse momentum muons; for this reason, the flavour physics measurements most readily accessible to the general purpose detectors are those in which the final state of the $B$ decay includes a muon.

LHCb [6] is a dedicated heavy-flavour experiment which will exploit the unprecedented quantity of $B$ hadrons produced at the LHC to overconstrain the CKM matrix and search for New Physics (NP) in the flavour sector. It will take data at a luminosity of $2 \cdot 10^{32} \mathrm{~cm}^{-2} \mathrm{~s}^{-1}$ with an $\eta$ coverage of $1.9<\eta<4.9$. The produced $B$ hadrons are highly boosted, which allows $\mathrm{LHCb}$ to achieve a typical lifetime resolution of $40 \mathrm{fs}$, allowing for precision measurements of time dependent $C P$ asymmetries in the neutral $B$ sector. LHCb will collect data at a rate of $2 \mathrm{kHz}$, using muon, hadron, and photon based triggers to select interesting events across a wide spectrum of final states.

\subsection{Data taking plans for 2009 and $\mathbf{2 0 1 0}$}

The LHC is due to restart in November 2009, and plans to take data through 2010, albeit at a reduced centre-of-mass energy. Several possible running scenarios exist and the precise conditions will be determined as more experience is gained with the machine.

\section{The decay $B_{s}^{0} \rightarrow \mu^{+} \mu^{-}$}

The rare, hitherto unobserved, decay $B_{s}^{0} \rightarrow$ $\mu^{+} \mu^{-}$has a predicted branching ratio of $(3.35 \pm 0.32) \cdot 10^{-9}$ in the SM [7], but this branching ratio is predicted to receive substantial corrections from many of the new particles which might be discovered at the LHC. Indeed, as seen in Figure 2, the branching ratio has discriminating power against a wide area of new physics parameter space. When this is combined with the fact that the final state contains only muons, making this mode accessible to ATLAS, CMS, and LHCb, it can be seen that $B_{s}^{0} \rightarrow \mu^{+} \mu^{-}$is an excellent candidate to observe of new physics at the LHC.

Since an absolute branching ratio measurement would be experimentally challenging, LHCb plans to measure the branching ratio of this decay relative to the control channels $B^{0} \rightarrow h^{+} h^{-}(h=$ $\pi, K)$ and $B^{+} \rightarrow J / \psi K^{+}$. A particular difficulty 


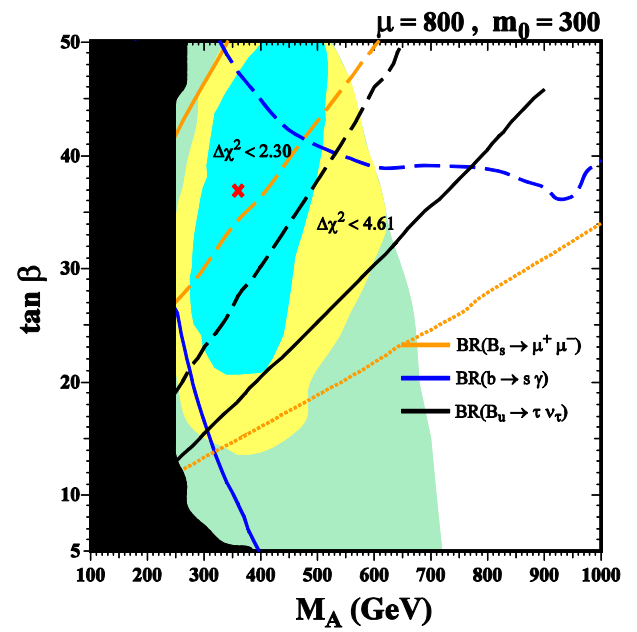

Figure 2. Best fit and $\chi^{2}$ contours in the plane $\left(M_{A}, \tan (\beta)\right)$ from the fit in [9] to several observables, including the anomalous magnetic moment of the muon. The orange lines indicate the excluded region when $B R\left(B_{s}^{0} \rightarrow \mu^{+} \mu^{-}\right)<10^{-7}$ (continuous), $2 \cdot 10^{-8}$ (dashed), or $5 \cdot 10^{-9}$ (dotted). Caption and figure taken from [8].

is the uncertainty on the $B_{s} / B_{d}$ production ratio, but it is hoped that new branching ratio measurements of $B_{s}$ decays by BELLE will go some way to solving this problem.

The simulated selection performance is limited by real muons from other $b$ and $c$ meson decays; neither decays in flight nor misidentification are a major source of background. Table 1 lists the signal and background yields for ATLAS, CMS, and LHCb; Figure 3 shows the LHCb discovery potential in this channel as a function of integrated luminosity.

\section{The decay $B_{d}^{0} \rightarrow K^{*} \mu^{+} \mu^{-}$}

The rare, flavour-changing neutral current, decay $B_{d}^{0} \rightarrow K^{*} \mu^{+} \mu^{-}$has several features which are highly sensitive to predictions made by new physics models [10]. The forward-backward asymmetry exhibits different features depending on which new physics model (or indeed the Stan-

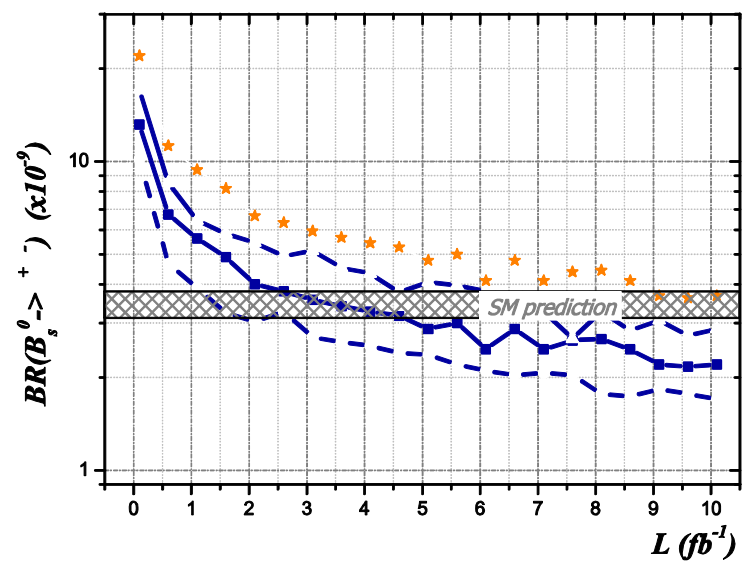

Figure 3. The LHCb discovery potential in the channel $B_{s}^{0} \mu^{+} \mu^{-}$as a function of integrated luminosity [8].

dard Model) is being considered, and the branching ratio also has some sensitivity to different new physics models as with $B_{s}^{0} \rightarrow \mu^{+} \mu^{-}$. Figures 4 and 5 show these dependences as a function of the dimuon mass $q^{2}$.

As the decay contains dimuons in the final state it is in principle accessible to ATLAS, CMS, and $\mathrm{LHCb}$; at the time of writing, however, only $\mathrm{LHCb}$ gives a publically available estimate of its performance in this channel and only it will therefore be considered. In common with the decay $B_{s}^{0} \rightarrow \mu^{+} \mu^{-}$, the main backgrounds come from real muons, with decays in flight and misidentified pions a negligible contribution. A particular difficulty of the analysis is the requirement that selection cuts be minimally biasing with respect to the angular distribution of the decay products. Figure 6 shows the estimated LHCb sensitivity to the forward-backward asymmetry spectrum after $2 \mathrm{fb}^{-1}$ of data taking. The crossing point sensitivity is estimated to be $\pm 0.5 \mathrm{GeV}^{2}$. 
Table 1

The expected signal and background yields (numbers of events) for ATLAS, CMS, and LHCb in the channel $B_{s}^{0} \mu^{+} \mu^{-}$[8]. The errors on the signal yields are negligible compared to the errors on the background.

\begin{tabular}{llll}
\hline Detector & Integrated Luminosity $\left(\mathrm{fb}^{-1}\right)$ & Signal & Background \\
\hline LHCb & 2 & 21 & $180_{-80}^{+140}$ \\
CMS & 10 & 6.1 & $14_{-14}^{+22}$ \\
ATLAS & 10 & 5.7 & $14_{-10}^{+13}$ \\
\hline
\end{tabular}

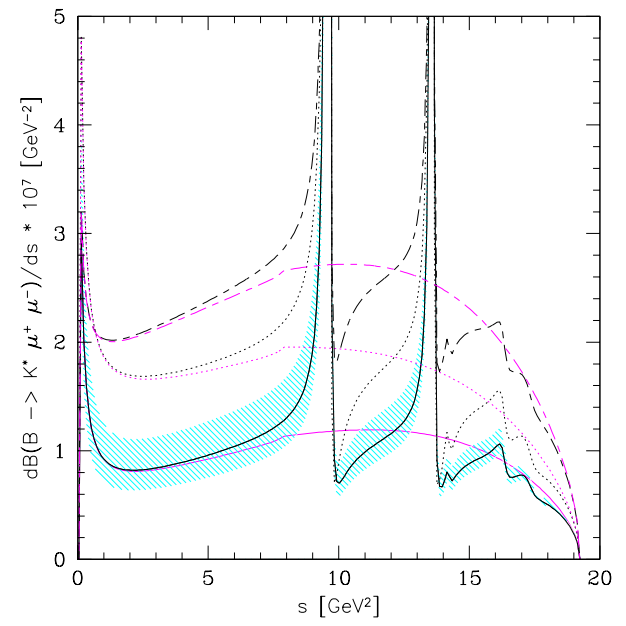

Figure 4. Theoretical $\mu^{+} \mu^{-}$mass distributions for $B_{d}^{0} \rightarrow K^{* 0} \mu^{+} \mu^{-}$decays in a number of models (solid line - SM; dotted line - SUGRA model; long-short dashed line - MIA-SUSY). The lower lines show the respective purely short-distance components. The shaded area around the SM line depicts the form factor-related uncertainties. Caption and figure taken from [8].

\section{The $B_{s}^{0}$ mixing phase $\phi_{s}$}

As explained in Section 1.1, recent CDF and D0 measurements of the $B_{s}^{0}$ mixing phase $\phi_{s}$ are in interesting tension with respect to the Standard Model prediction. The LHC experiments aim to resolve this tension by measuring $\phi_{s}$ with a $3 \sigma$ precision even if $\phi_{s}$ is at its Standard Model value.

Although the $B_{s}^{0}$ mixing phase $\phi_{s}$ is very small in the $\mathrm{SM}$, it can receive sizable NP contribu-

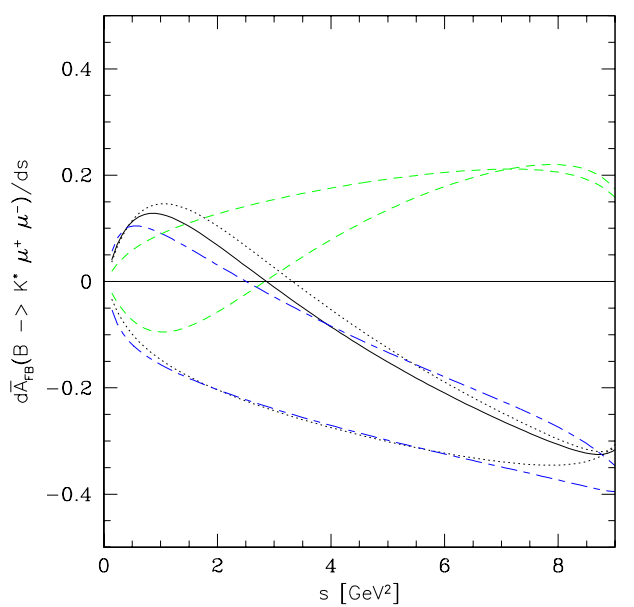

Figure 5. Theoretical muon forward-backward asymmetry for $B_{d}^{0} \rightarrow K^{* 0} \mu^{+} \mu^{-}$in a number of models (solid line - SM; dotted line SUGRA model; long-short dashed line - MIASUSY; dashed line - MIA-SUSY with $C_{10}>0$ ). The upper (lower) lines have $C_{7}^{\text {eff }}<0\left(C_{7}^{\text {eff }}>\right.$ $0)$. Caption and figure taken from [8].

tions through box diagrams involving top quark exchange. The golden mode for measuring $\phi_{s}$ is $B_{s}^{0} \rightarrow J / \psi \phi$. ATLAS, CMS, and LHCb all aim to study this channel, however at the time of writing only LHCb has published estimates of its sensitivity to $\phi_{s}$ and therefore the remainder of this Section will refer to $\mathrm{LHCb}$ alone. Table 2 shows the expected signal yields and purities for the three detectors. Note that the LHCb background levels are expected to be significantly higher because $\mathrm{LHCb}$ uses a lifetime unbiased event selection; 
Table 2

The expected signal yields and purities for ATLAS, CMS, and LHCb in the channel $B_{s}^{0} \rightarrow J / \psi \phi[8]$.

\begin{tabular}{llll}
\hline Detector & Integrated Luminosity $\left(\mathrm{fb}^{-1}\right)$ & Signal Yield & $\mathrm{B} / \mathrm{S}$ \\
\hline LHCb & 2 & $117 \mathrm{k}$ & 2.1 \\
$\mathrm{CMS}$ & 10 & $109 \mathrm{k}$ & 0.3 \\
ATLAS & 10 & $80 \mathrm{k}$ & 0.3 \\
\hline
\end{tabular}

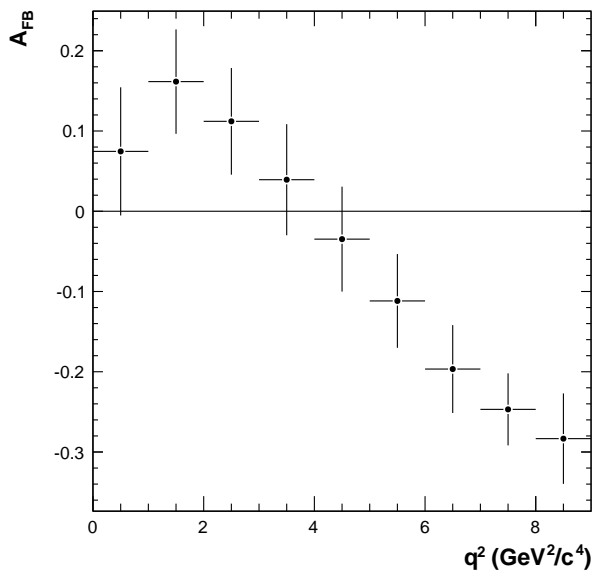

Figure 6. Estimated LHCb sensitivity to the muon forward-backward asymmetry spectrum in $B_{d}^{0} \rightarrow K^{* 0} \mu^{+} \mu^{-}$after $2 \mathrm{fb}^{-1}$ of data taking [8].

such a selection results in a signal sample with a higher per event sensitivity to the parameter $\phi_{s}$.

The measurement of $\phi_{s}$ from the time dependent decay rate asymmetries is complicated by the fact that $B_{s}^{0} \rightarrow J / \psi \phi$ is not a pure $\mathrm{CP}$ mode. CP-even and CP-odd contributions can be separated by studying their distributions in the transversity angle. Figure 7 shows the definition of the three angles $\theta, \phi, \psi$ which describe the system, while Figure 8 shows the separation of the CP-even and CP-odd components in the angle $\theta$.

LHCb estimates that a precision of $0.03 \mathrm{rad}$ on $\phi_{s}$ can be reached with $2 f b^{-1}$ of data taking. After $10 \mathrm{fb}^{-1}$ of data taking, LHCb will have $>3 \sigma$

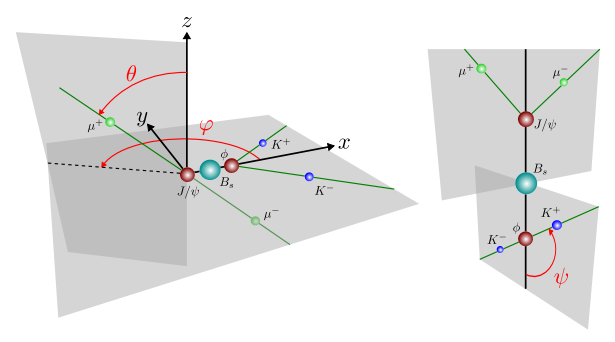

Figure 7. The angles describing the decay $B_{s}^{0} \rightarrow$ $J / \psi \phi .[8]$

evidence of a non-zero $\phi_{s}$ even if no new physics is discovered. In addition, other CP-specific final states will be measured[11], leading to a small improvement in the overall precision.

\section{The CKM angle $\gamma$}

The CKM angle $\gamma$ is still one of the least well known parameters of the CKM triangle, and a precision measurement of $\gamma$ will be crucial to the interpretation of any New Physics which may manifest in the flavour sector. Because the decay modes sensitive to $\gamma$ have purely hadronic final states, LHCb is the only one of the LHC experiments which is able to measure $\gamma$.

5.1. Measuring $\gamma$ from $B_{d, s}^{0} \rightarrow h^{+} h^{-}$decays The $B_{d, s}^{0} \rightarrow h^{+} h^{-}$family of decays, where $h$ stands for a $\pi$ or $K$ meson, have decay rates with non-negligible contributions from penguin diagrams, making them sensitive to NP. The pos- 


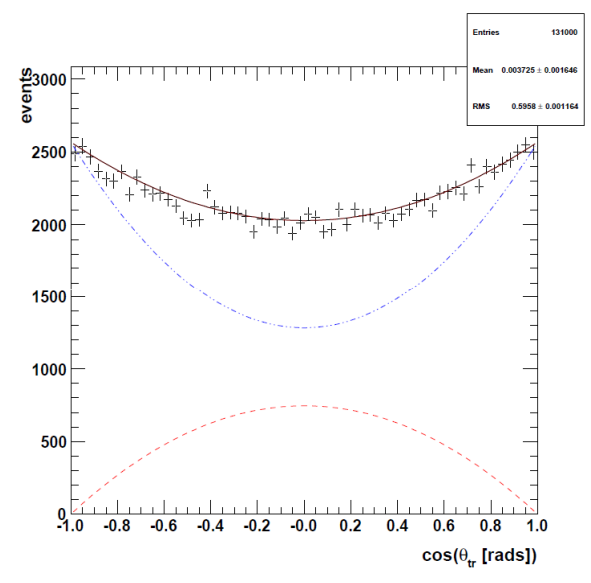

Figure 8. A graphic illustrating the separation of the CP-even (blue, dash-dotted) and CP-odd (red, dashed) components in the channel $B_{s}^{0} \rightarrow$ $J / \psi \phi$ according to the transversity angle $\theta$. [12]

sible Feynmann diagrams for these decays are shown in Figure 9.

The dependence on $\gamma$ comes from the timedependent $\mathrm{CP}$ asymmetries in the $B_{s}^{0} \rightarrow K^{+} K^{-}$ and $B_{d}^{0} \rightarrow \pi^{+} \pi^{-}$decays, given by

$A_{C P}(t)=\frac{A_{d i r} \cos (\Delta m t)+A_{m i x} \cos (\Delta m t)}{\cosh (\Delta \Gamma t / 2)+A_{\Delta \Gamma} \sinh (\Delta \Gamma t / 2)}$.

The parameters $A_{d i r}, A_{m i x}$ are the direct and mixing induced $\mathrm{CP}$ asymmetry terms and depend on $\gamma$. However, these asymmetry terms also depend on the amplitude ratio of the penguin and tree decay diagrams $d_{h h} e^{i \theta_{h h}}$, as well as the mixing phases $\phi_{d}$ and $\phi_{s}$. Since there are four asymmetries and seven unknown parameters, it is necessary to employ U-spin symmetry, which leads to $d_{\pi \pi} \equiv d_{K K}$ and $\theta_{\pi \pi} \equiv \theta_{K K}$. This allows $\gamma$ to be measured when combined with external constraints on $\phi_{d}$ and $\phi_{s}$. It is estimated $[8,13]$ that this method allows $\gamma$ to be determined, allowing for a $20 \%$ level of U-spin breaking, with a precision of $7^{\circ}$ after $2 \mathrm{fb}^{-1}$ of LHCb data taking. The Bayesian likelihood for $\gamma$ corresponding to this amount of data can be seen in Figure 10.

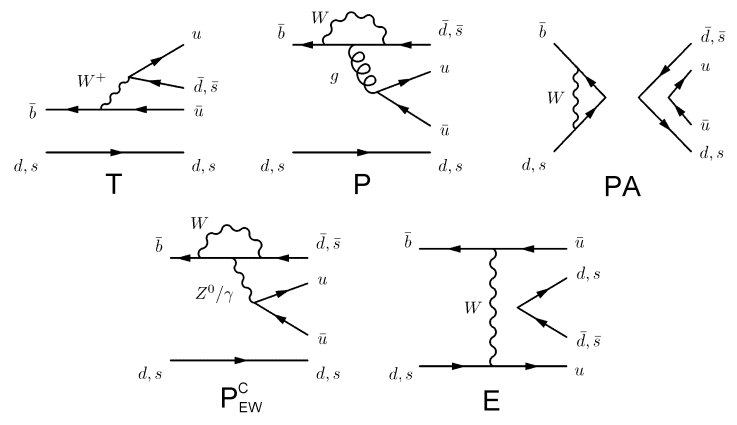

Figure 9. An example of possible Feynmann diagrams for the $B_{d, s}^{0} \rightarrow h^{+} h^{-}$family of decays [8].

5.2. Measuring $\gamma$ from $B^{ \pm} \rightarrow D^{0} K^{ \pm}$decays

The charmed decays of charged $B$ mesons proceed through tree-level diagrams, and enable a direct SM measurement of $\gamma$. First measurements of this kind have already been made at the B factories[14], and are the dominant inputs to the global average on $\gamma$ quoted is Section 1.1.

Different strategies exist for measuring $\gamma$, depending on the final state into which the $D^{0}$ decays. In the GLW[15,16] strategy, the $D^{0}$ decays into a $\mathrm{CP}$ eigenstate, and the sensitivity to $\gamma$ comes from the interference between dominant and doubly colour-suppressed decays. The ADS[17] strategy combines colour-suppressed B decays with colour-favoured $\mathrm{D}$ decays (and vice versa), thus increasing the interference effects. The GGSZ[18] strategy uses a Dalitz analysis of $D^{0} \rightarrow K_{S} \pi \pi$ decays to extract $\gamma$ together with the strong phases in the $D^{0}$ decay. The sensitivity to $\gamma$ in this case comes from the differences in the Dalitz plot for $B^{+}$and $B^{-}$decays as can be seen in Figure 11.

\subsection{Measuring $\gamma$ from $B_{s, d}^{0} \rightarrow D_{s, d}^{ \pm}\left(K^{\mp}, \pi^{\mp}\right)$ decays}

The time-dependent $\mathrm{CP}$ asymmetries in the tree level decays $B_{s}^{0} \rightarrow D_{s}^{ \pm} K^{\mp}$ and $B_{d}^{0} \rightarrow D^{ \pm} \pi^{\mp}$ can be used to measure the SM value of $\gamma+\phi_{s, d}$. Together with the measurement of $\gamma$ from charged $B$ decays, they will provide a baseline SM measurement of $\gamma$; this will allow any NP effects in 


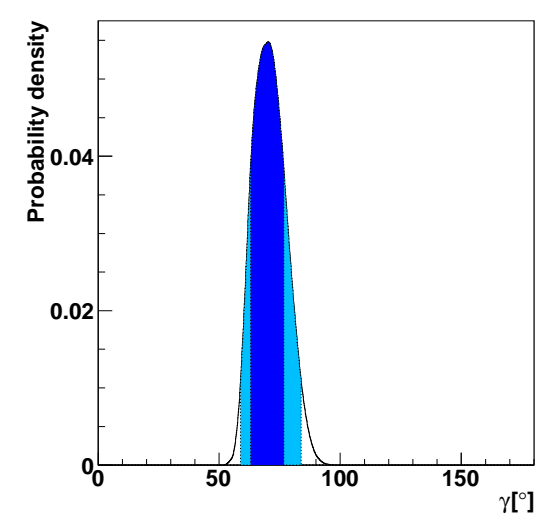

Figure 10. The likelihood for $\gamma$ after $2 f b^{-1}$ of LHCb data taking in the $B_{d, s}^{0} \rightarrow h^{+} h^{-}$family of decays [8]. The dark blue region corresponds to the $68 \%$ confidence interval, the light blue to the $95 \%$ confidence interval.

the measurement of $\gamma$ from $B^{0} \rightarrow h^{+} h^{-}$decays to be constrained.

The value of $\gamma$ extracted from these asymmetries in principle suffers from an eightfold ambiguity. The expected sizable lifetime difference $\Delta \Gamma_{s}$ in the $B_{s}^{0}$ system gives access to two additional observables which reduce the eightfold ambiguity to a twofold one when the flavour specific $B_{s}^{0} \rightarrow D_{s}^{-} \pi^{+}$decays are used in a simultaneous fit to constrain $\Delta \Gamma_{s}$ and $\Delta m_{s}$. An example fit to the $B_{s}^{0}$ oscillations is shown in Figure 12.

In contrast with the $B_{s}^{0}$ mesons, no sizable lifetime difference is expected in the $B_{d}^{0}$ system and the ambiguities remain. In addition, the interference term in the $B_{d}^{0}$ decays is too small to fit directly from the $\mathrm{CP}$ asymmetries and will have to be externally constrained. Both of these problems can be solved by relating the decays $B_{s}^{0} \rightarrow D_{s}^{ \pm} K^{\mp}$ and $B_{d}^{0} \rightarrow D^{ \pm} \pi^{\mp}$ through U-spin symmetry[2123], allowing for a fully unambiguous measurement of $\gamma$. The decay $B_{d}^{0} \rightarrow D^{ \pm} \pi^{\mp}$ has another important role to play in these measurements; as the most abundant decay mode of this type, it will be used to calibrate the selection and anal-
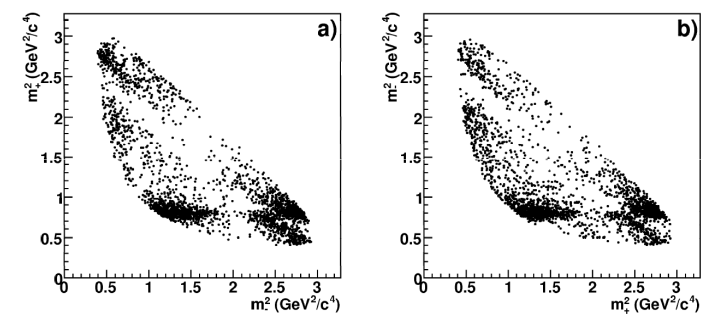

Figure 11. The Dalitz plots for $B^{ \pm} \rightarrow D^{0} K^{ \pm}$ decays where the $D^{0}$ decays to $K_{S} \pi \pi$. Figure (a) is for $B^{+}$and figure (b) for $B^{-}$mesons. The differences observable in the top left of the two plots provide the sensitivity to $\gamma$ [19].

ysis of the other decays such as $B_{s}^{0} \rightarrow D_{s}^{ \pm} K^{\mp}$. To illustrate this, Figure 13 shows the mass peak reconstructed on simulated $b \bar{b}$ events after $300 \mathrm{~s}$ of nominal LHCb data taking.

\subsection{Combined sensitivity to $\gamma$ from charmed B decays}

The time-dependent and direct $\mathrm{CP}$ violation measurements of $\gamma$ listed in Sections 5.2 and 5.3 can be combined to give an overall tree-level precision on $\gamma$. It is estimated [8] that the achievable precision will be (4-5) after $2 \mathrm{fb}^{-1}$ of data taking, or $(8-10)^{\circ}$ after $500 \mathrm{pb}^{-1}$ of data taking.

\section{Measurements of radiative decays}

The family of radiative penguin decays $B \rightarrow$ $X \gamma$, examples of which include the decays $B_{s}^{0} \rightarrow$ $\phi \gamma ; B^{0} \rightarrow K^{*} \gamma ;$ and $B^{+} \rightarrow \phi K^{+} \gamma$, are sensitive to new physics through the appearance of new virtual particles in the loop. Moreover, many different measurements offer ways to probe this sensitivity. Early on in the LHC's data taking, interesting measurements include the direct CP asymmetry in the decay $B^{0} \rightarrow K^{*} \gamma$ and the ratio of $B^{0} \rightarrow K^{*} \gamma$ and $B_{s}^{0} \rightarrow \phi \gamma$ decay rates. In the long term, photon polarization and measurements through time dependent $\mathrm{CP}$ violation in $B_{s}^{0} \rightarrow \phi \gamma$ offer very sensitive probes of new physics. Table 3 lists the estimated yields and purities of these 
Table 3

The expected signal yields and purities for LHCb in the $B \rightarrow X \gamma$ family of channels [8].

\begin{tabular}{lll}
\hline Channel & Signal Yield $\left(2 \mathrm{fb}^{-1}\right)$ & $\mathrm{B} / \mathrm{S}$ \\
\hline$B^{0} \rightarrow K^{*} \gamma$ & $70 \mathrm{k}$ & 0.6 \\
$B_{s}^{0} \rightarrow \phi \gamma$ & $11 \mathrm{k}$ & $<0.6$ \\
$B^{+} \rightarrow \phi K^{+} \gamma$ & $7 \mathrm{k}$ & $<2.0$ \\
\hline
\end{tabular}

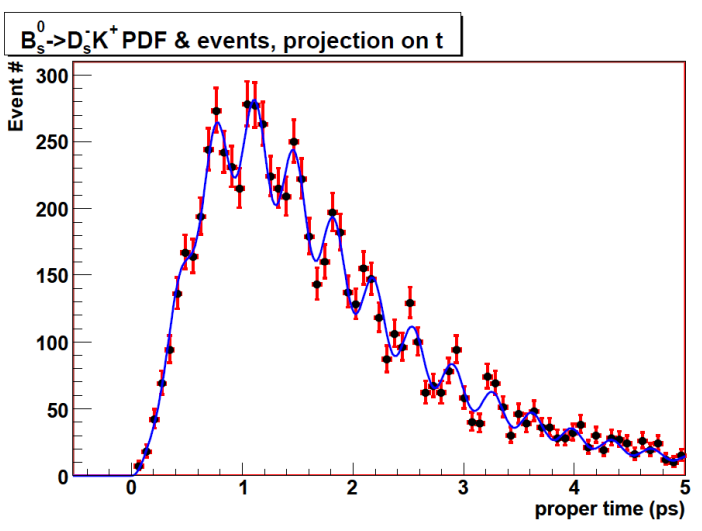

Figure 12. An example fit to the $B_{s}^{0}$ oscillations in the $B_{s}^{0} \rightarrow D_{s}^{-} K^{+}$channel $[20]$.

channels at LHCb.

\section{Conclusion}

The unprecedented centre-of-mass energies achievable at the LHC are usually thought of as allowing new particles to be directly produced in the proton-proton interaction, but they will also result in a tremendous cross-section for $b \bar{b}$ events and hence potential for studies in heavy flavour physics. This contribution has shown how both the general-purpose detectors, ATLAS and CMS, and the LHC's dedicated heavy-flavour detector, $\mathrm{LHCb}$, will exploit this cross section to probe for new physics in the $B$ sector, both through studies of rare decay processes such as $B_{s}^{0} \rightarrow \mu^{+} \mu^{-}$as well as precision measurements of $\mathrm{CP}$ violation in hadronic and radiative decay modes, in particular at LHCb. It would be amiss to conclude, however, without mentioning the LHC's potential to

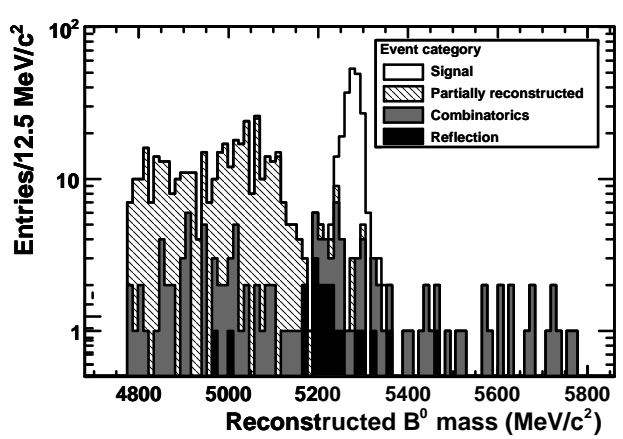

Figure 13. The $B_{d}^{0} \rightarrow D^{ \pm} \pi^{\mp}$ mass peak reconstructed on simulated $b \bar{b}$ events after 300 seconds of nominal LHCb data taking [24].

perform charm physics. If the cross-section for $b \bar{b}$ events is high at the LHC, the cross-section for $c \bar{c}$ events is higher still, and $\mathrm{LHCb}$ is studying triggers and selections for charm decays in order to probe for $\mathrm{CP}$ violation in the charm sector. It is a historical fact that experiments often end up being remember for something other than their design purpose; it would be rather ironic if the LHC is remembered for the precision measurements of $\mathrm{CP}$ violation in the decays of charmed mesons.

\section{Acknowledements}

The author would like to thank the organizers of the BSM-LHC workshop for the opportunity to present this work, and Paula Eerola, Samira Hassani, Jim Olsen, and Chariclia Petridou for their assistance in understanding the ATLAS and CMS B-physics programmes. 


\section{REFERENCES}

1. CKMfitter Group (J. Charles et al.), Eur. Phys. J. C41, 1-131 (2005). Updated results and plots available at: http://ckmfitter.in2p3.fr

2. Combination of D0 and CDF Results on $\Delta \Gamma_{s}$ and the CP-Violating Phase $\beta_{s}^{J / \psi \phi}$, CDF/PHYS/BOTTOM/CDFR/9787, D0 Note 5928-CONF, 2009

3. LHC Study Group, CERN/AC/90-05(LHC) (1995).

4. G. Aad et al., CERN-OPEN-2008-020 (2008) [arXiv:0901.0512 [hep-ex]].

5. A. de Roeck et al., CERN/LHCC 2006-021 (2006).

6. The LHCb Collaboration, The LHCb detector at LHC, JINST 3 S08005 (2008).

7. M. Blanke, A.J. Buras, D. Guadagnoli and C. Tarantino, arXiv:hep-ph/0604057 (2006). .

8. The LHCb Collaboration, The LHCb Analysis Roadmap, in preparation.

9. J. Ellis, T. Hahn, S. Heinemeyer, K.A. Olive and G. Weiglein, arXiv:hep-ph/0709.0098 (2007).

10. A. Ali, T. Mannel and T. Morozumi, Forward backward asymmetry of dilepton angular distribution in the decay $b \rightarrow s l^{+} l^{-}$., Phys.Lett.B273 505,1991

11. D. Volyanskyy and J. Van Tilburg, CERNLHCb-2007-027 (2007).

12. P. Clarke and C. McLean, CERN-LHCb2007-101 (2007).

13. A. Carbone, J. Nardulli, S. Pennazzi, A. Sarti, and V. Vagnoni, CERN-LHCb-2007059 (2007).

14. V. Tisserand, Belle and BaBar Collaborations, Proceedings of 5th Flavor Physics and CP Violation Conference (FPCP 2007), Bled, Slovenia, 9 (2007).

15. M. Gronau and D. London, Phys. Lett. B253, 483-488 (1991).

16. M. Gronau and D. Wyler, Phys. Lett. B265, 172-176 (1991).

17. D. Atwood, I. Dunietz, and A. Soni, Phys. Rev. Lett. 78, 3257-3260 (1997).

18. A. Giri, Y. Grossman, A. Soffer, and J. Zupan, Phys. Rev. D68, 054018 (2003).
19. J. Libby, L. Martin, CERN-LHCb-2007-142 (2007).

20. S. Cohen, M. Merk, and E. Rodrigues, CERN-LHCb-2007-041 (2007).

21. R. Fleischer, Nucl. Phys. B671, 459-482 (2003).

22. G. Wilkinson, CERN-LHCb-2005-036 (2005).

23. V. V. Gligorov, CERN-LHCb-2007-044 (2007).

24. V. V. Gligorov, CERN-LHCb-2009-003 (2009). 